Three-dimensional hydration layer mapping on the (10.4) surface of calcite using amplitude modulation atomic force microscopy

This content has been downloaded from IOPscience. Please scroll down to see the full text. 2014 Nanotechnology 25335703

(http://iopscience.iop.org/0957-4484/25/33/335703)

View the table of contents for this issue, or go to the journal homepage for more

Download details:

IP Address: 134.93.201.146

This content was downloaded on 26/08/2014 at 12:11

Please note that terms and conditions apply. 


\title{
Three-dimensional hydration layer mapping on the (10.4) surface of calcite using amplitude modulation atomic force microscopy
}

\author{
Christoph Marutschke ${ }^{1}$, Deron Walters ${ }^{2}$, Jason Cleveland ${ }^{2}$, Ilka Hermes ${ }^{1}$, \\ Ralf Bechstein ${ }^{1}$ and Angelika Kühnle ${ }^{1}$ \\ ${ }^{1}$ Institut für Physikalische Chemie, Johannes Gutenberg Universität Mainz, Duesbergweg 10-14, D-55099 \\ Mainz, Germany \\ ${ }^{2}$ Asylum Research an Oxford Instruments Company, Santa Barbara, CA 93117, USA \\ E-mail: kuehnle@uni-mainz.de
}

Received 20 December 2013, revised 11 March 2014

Accepted for publication 3 April 2014

Published 30 July 2014

\begin{abstract}
Calcite, the most stable modification of calcium carbonate, is a major mineral in nature. It is, therefore, highly relevant in a broad range of fields such as biomineralization, sea water desalination and oil production. Knowledge of the surface structure and reactivity of the most stable cleavage plane, calcite (10.4), is pivotal for understanding the role of calcite in these diverse areas. Given the fact that most biological processes and technical applications take place in an aqueous environment, perhaps the most basic_-yet decisive-question addresses the interaction of water molecules with the calcite (10.4) surface. In this work, amplitude modulation atomic force microscopy is used for three-dimensional (3D) mapping of the surface structure and the hydration layers above the surface. An easy-to-use scanning protocol is implemented for collecting reliable 3D data. We carefully discuss a comprehensible criterion for identifying the solid-liquid interface within our data. In our data three hydration layers form a characteristic pattern that is commensurate with the underlying calcite surface.
\end{abstract}

S Online supplementary data available from stacks.iop.org/NANO/25/335703/mmedia

Keywords: AFM, 3D mapping, hydration layer, solid-liquid interface, calcite

(Some figures may appear in colour only in the online journal)

\section{Introduction}

Calcite, the most stable modification of calcium carbonate $\left(\mathrm{CaCO}_{3}\right)$, has been studied extensively due to its importance in various fields, especially in biomineralization [1]. The (10.4) surface as the most stable cleavage plane has, consequently, attracted significant research interest with an emphasis on understanding its morphology and reactivity. The surface unit cell is rectangular with dimensions of $8.1 \AA \times 5.0 \AA$ as shown in figure 1 . It contains two carbonate groups, which are rotated with respect to the surface normal and with respect to each other. The detailed surface structure has been elucidated using atomic force microscopy (AFM) operated in air [2] and under aqueous conditions [2-6]. True atomic resolution on this surface in a liquid environment has been achieved recently using a modified AFM operated in the frequency modulation (FM) mode [7].

A number of studies have addressed calcite growth [8-11] and etch pit formation [12] in oversaturated and undersaturated $\mathrm{CaCO}_{3}$ solution, respectively. Surface morphology changes due to the presence of organic molecules $[10,12]$ have been investigated. Recently, the $\mathrm{pH}$-dependent binding of an organic molecule to the calcite surface has been revealed by means of FM-AFM at the molecular level [13]. 


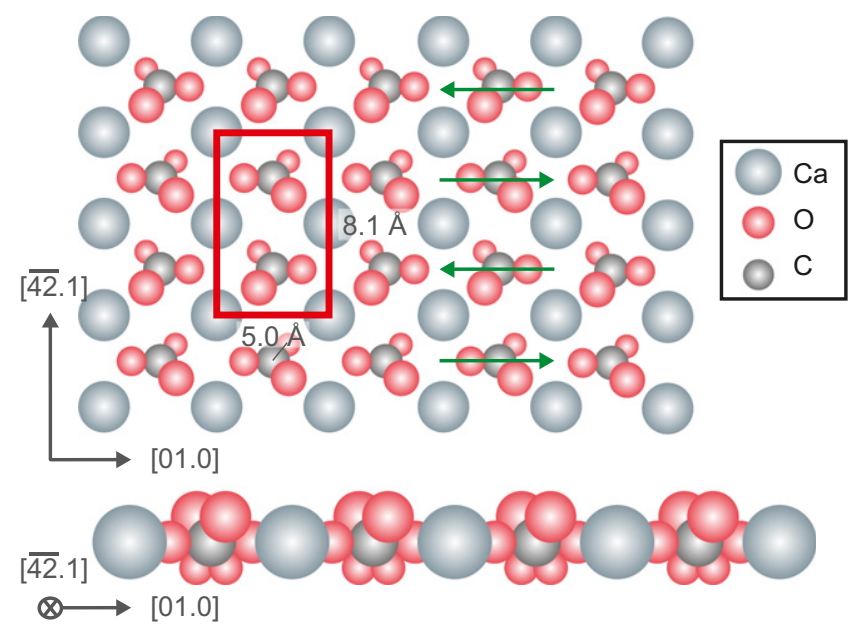

Figure 1. Sketch of the (10.4) surface of calcite with the rectangular unit cell. The green arrows indicate the different orientation of the carbonate groups with respect to each other along the [01.0] direction.

The interaction of water with the calcite surface has been studied experimentally, employing x-ray scattering [14-16] and, only very recently, using FM-AFM. Based on FM-AFM data, two groups have identified distinct water patterns above calcite. Imada et al have reported a 'checkerboard' pattern along the [01.0] direction [17]. Kobayashi et al have seen 'diamond-like' and 'line-shaped' patterns along [01.0] [18], which are tentatively ascribed to the two different orientations of the carbonate groups on the surface (compare green arrows in figure 1). Among the theoretical studies addressing the water-calcite interaction [19-22], especially the work of Reischl et al is interesting since it takes the AFM tip into account [22].

The aforementioned experimental and theoretical studies suggest that water forms well-ordered structures above the calcite (10.4) surface and at least three hydration layers have been identified. It is believed that the first water layer is adsorbed with its oxygen atoms on top of the surface calcium ions, while the second water layer is ascribed to water molecules forming hydrogen bonds with the protruding oxygen atoms of the carbonate groups. The third hydration layer has been considered to be only weakly bound with less lateral order compared to the first and second hydration layer.

While standard AFM imaging is capable of atomic resolution on a planar surface, a three-dimensional (3D) mapping technique is required to study the formation of solvent molecules above the surface. The development of 3D mapping techniques has attracted a lot of attention in the AFM community in the last years. Two different data acquisition protocols can be distinguished, i.e., the 'curve-bycurve' approach [23], performing a set of force curves, and the 'layer-by-layer' approach, imaging the surface at different tip-sample distances [24]. On calcite (10.4), the first 3D map under UHV conditions has been presented in 2011 by our group [25]. Mapping experiments in water have been performed on pristine surfaces such as mica [26], $\alpha-\mathrm{Al}_{2} \mathrm{O}_{3}$ [27] and graphite [28]. These studies have later been extended to molecular crystals [29], layers of organic molecules [30], biological systems such as lipid layers [31, 32], and longchain alkanes as solvent $[33,34]$. The first $3 \mathrm{D}$ data set in water showing hydration layers has been presented by Fukuma et al on mica [35].

To our knowledge, all fundamental work on hydration layer mapping has been done by means of FM-AFM. FM$\mathrm{AFM}$ is a challenging technique that requires three feedback loops to control the phase, the amplitude and the tip-sample distance while the frequency of the cantilever oscillation is precisely measured. Here, we present an easy-to-use and reliable method to perform 3D data collection employing amplitude modulation AFM (AM-AFM). Compared to FMAFM, AM-AFM is easier to use since only one feedback loop is required to control the tip-sample distance while the amplitude and the phase are directly used as the imaging signals. For the ease of implementation, we decided to use a commercially available AFM. In this paper, we report on the implementation of the 3D scanning protocol, and we demonstrate the performance of our set-up by mapping the hydration layers above the calcite (10.4) surface.

\section{3D data acquisition protocol}

We implemented the 3D data acquisition protocol on a Cypher AFM from Asylum Research (Oxford Instruments, UK) equipped with a standard scanner and an open liquid cell. The instrument is operated using the data evaluation software Igor Pro (WaveMetrics, USA) that offers an interface to write own control routines. We integrated the $3 \mathrm{D}$ protocol into the standard scanning routine by applying an additional, sinusoidal modulation to the z-piezo voltage, causing a modulation of the tip-sample distance ('curve-bycurve' approach) [35]. Due to the sinusoidal movement of the z-piezo, more data points are acquired around the turning points. No threshold or abort condition for the oscillation amplitude or the phase is implemented in our routine. Hence, the volume of the 3D map is precisely determined by two parameters, i.e., the average tip-sample distance and the modulation range as shown in figure 2. The average tipsample distance is determined by the oscillation amplitude setpoint expressed as fraction of the free oscillation amplitude. A small amplitude setpoint corresponds to a small tipsample distance, while a large setpoint means a large tipsample separation. The amplitude setpoint remains unchanged during the modulation of the z-piezo voltage. The distancefeedback loop operates only very slowly during the data acquisition, i.e. too slow to compensate the modulation but fast enough to compensate $\mathrm{z}$-drift effects and tilt caused by misalignment of the sample. Before the data acquisition starts, our routine adjusts the phase shift between excitation and cantilever response to $90^{\circ}$, which allows for easy comparison of data acquired in different experiments.

A major difficulty when performing AFM in liquids is the so-called 'forest of peaks' [36]. This term refers to the various additional peaks in the resonance curve, which are caused by the standard piezo excitation of the entire liquid 
(a)


(b)

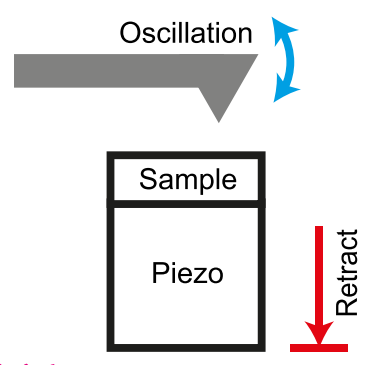

Average

tip-sample

distance

Figure 2. Illustration of the implementation of the 3D data acquisition protocol into the normal scan routine. During the retrace movement (a), the average tip-sample distance is determined by the oscillation amplitude setpoint. The z-piezo voltage is modulated and the data collection takes place. In the trace movement (b), the sample is retracted and a feedback loop adjusts the excitation to keep the free amplitude of the cantilever constant during the measurement.

cell. These additional resonances constantly change their frequency upon tiny changes in, e.g., the amount of liquid in the cell. The free amplitude is, therefore, subject to changes caused by this drifting 'forest of peaks', which superimposes with the stable resonance of the cantilever. To compensate for this effect, the excitation needs to be adjusted regularly to keep the free amplitude constant. We implemented a feedback loop, that adjusts the excitation amplitude during the 3D data acquisition. The amplitude and phase data are only collected during one half of each scan line (usually the retrace movement) as shown in figure 2(a). Here, the sample is in close vicinity to the tip and the z-piezo modulation voltage is applied. In the second half of each scan line (usually the trace movement), the sample is pulled away from the tip, no zmodulation takes place and no 3D mapping is done (see figure 2(b)). Instead, the excitation feedback adjusts the excitation amplitude to maintain a constant free amplitude throughout the entire measurement. The technical implementation of the excitation feedback consists of a lock-in for measuring the free amplitude of the cantilever and a built-in PIDS feedback loop of the Cypher AFM that is used for regulating the excitation amplitude. During the adjustment procedure the distance feedback loop is switched off. The extent of necessary adjustment of the excitation amplitude depends crucially on the shape of the 'forest of peaks' and on the drift conditions of the specific measurement, both beeing unpredictable. Without compensation, we usually observe that the free amplitude is subject to significant changes on the timescale required for one 3D map, i.e. 30 s. To illustrate the effect of the drifting 'forest of peaks' we show a typical measurement of the free amplitude at constant excitation in the supplementary information (figure S1).

Our 3D data acquisition protocol is capable of capturing the four relevant signals, i.e., amplitude, phase, static cantilever deflection, and the output of the distance feedback loop.

The static deflection signal is obtained by applying a $25 \mathrm{kHz}$ low-pass filter to the photo-diode signal (dynamic deflection signal).

To suppress drift effects, the experiment needs to be performed with very high scan rates compared to those typically applied in UHV. In our set-up, scan rates between $2 \mathrm{~Hz}$ and $4 \mathrm{~Hz}$ proved to be very effective. The frequency of the tip-sample distance modulation has to be considerably higher than the scan rate and significantly lower than the oscillation frequency of the cantilever. We used modulation frequencies between $500 \mathrm{~Hz}$ and $750 \mathrm{~Hz}$ in our experiments. Using the full speed of our electronics, we can sample the signal from the photo-diode at a rate of $50 \mathrm{kHz}$. With this sample rate, a map of $96 \times 240 \times 68$ pixel $(x \times y \times z)$, which includes over-scanning and free-amplitude adjustment, requires approximately $30 \mathrm{~s}$.

\section{Experimental set-up}

For the measurements, we used Arrow UHV cantilevers (Nanoworld, Switzerland) with a high resonance frequency in liquids of around $350 \mathrm{kHz}$. The quality factor $(\mathrm{Q})$ of the cantilevers was determined by a harmonic fit to the thermal spectra. We measured $Q$ values between 4 and 7 in aqueous solution. Values for the spring constant (of the order of $4 \frac{\mathrm{N}}{\mathrm{m}}$ ) and for the sensitivity of the photo-diode were determined by the thermal noise method [37] and by static deflection force curves. These figures were measured from time to time for individual cantilevers. For cantilevers originating from the same batch, no large deviations were observed. We used an average sensitivity of $22 \frac{n m}{V}$ to calculate the amplitude values for the data presented in this article. Using an average value increases the error in the specification of the oscillation amplitude, however, as we are interested in a qualitative evaluation of the data, this is of minor importance. As free amplitude we usually used values between $0.3 \mathrm{~nm}$ and $1.0 \mathrm{~nm}$. Typical amplitude setpoints were between 0.7 and 0.9 , i.e. $70 \%-90 \%$ of the free amplitude. As z-modulation range we chose values between $\pm 0.5 \mathrm{~nm}$ and $\pm 1.0 \mathrm{~nm}$.

All measurements shown here were acquired in an open cell with a highly oversaturated solution of $\mathrm{CaCO}_{3}(2 \mathrm{mM})$ to avoid dissolution of the calcite sample during data acquisition. The ionic solution was also used to control the Debye length of the hydration layers, which is a common approach in liquid AFM experiments [26]. The solution was prepared by mixing corresponding quantities of sodium bicarbonate and calcium chloride with Milli-Q water (Millipore GmbH, Germany). Calcite (Iceland Spar) was purchased from Wards Science (USA). The crystals were freshly cleaved before each measurement and immediately immersed into the $\mathrm{CaCO}_{3}$ solution. The actual temperature of the scan head during data collection was recorded for each experiment. 

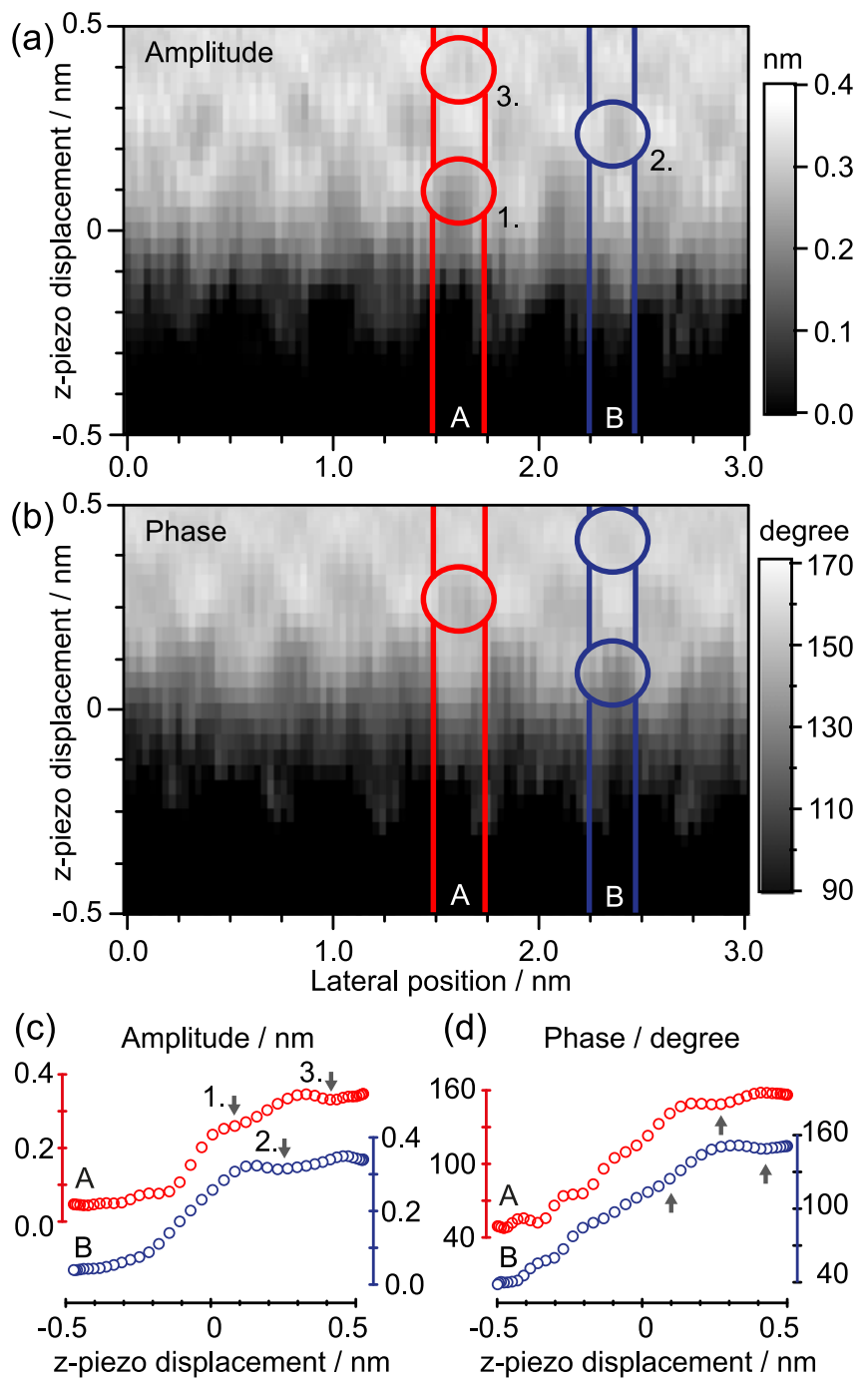

Figure 3. Oscillation amplitude (a) and phase (b) 2D AM-AFM slice along the [01.0] direction of calcite (10.4). Two regions of interest are marked with red (A) and blue (B) boxes. Here oscillation amplitude (c) and phase (d) 1D data was extracted via averaging over the indicated areas. Modulations within the signals are marked with circles (a), (b) and arrows (c), (d).

\section{Results and discussion}

To demonstrate the capabilities of our data acquisition routine we measured 3D AM-AFM maps in aqueous solution over calcite (10.4). We extracted 2D data slices along high-symmetry surface directions from these 3D data sets. In figures 3 (a), (b), a representative slice along the [01.0] direction is shown. The corresponding 3D map was acquired at a temperature of $299 \mathrm{~K}$, with a free amplitude of $0.4 \mathrm{~nm}$, an amplitude setpoint of 0.7 (comparable to previous studies [17]) and a modulation range of $\pm 0.5 \mathrm{~nm}$. Although we took great care to align the crystal such that the fast scan direction coincides with the [01.0] direction, slight misalignments always remain due to, e.g., drift effects. Here, we benefit from the fact that 3D data was collected, as it allows for cutting the desired slice from the data volume using the Bresenham Line Algorithm [38]. Therefore, 3D data collection has a clear advantage as compared to $2 \mathrm{D}$ data acquisition. The slice in figures 3(a), (b) shows data obtained during approach, i.e., when the sample moves towards the cantilever. The retract data (not shown) does not differ notably from the approach data.

As can be seen in figure 3, we achieved detailed resolution both laterally and perpendicular to the surface. When inspecting the amplitude signal (figure 3(a)), three distinct minima can be identified above the surface in two site-specific areas (A and B). These minima are periodically repeated parallel to the surface, which results in the characteristic checkerboard pattern as previously observed by FM-AFM $[17,18]$. A similar pattern is visible in the phase signal (figure 3(b)), however, here maxima are observed where the amplitude signal shows minima and vice versa.

Contrast formation in AFM is generally very complicated since it strongly depends on the complex interaction between sample, cantilever tip and the surrounding water. As usual for AFM experiments in liquids, the tip termination is crucial for the contrast formation but impossible to be controlled precisely. This is especially important for the interaction between the water molecules and the tip [39]. In repetitions of the experiment presented in figures 3(a), (b), we found the checkerboard pattern to be more or less pronounced depending on the (unknown) tip termination and the experimental settings. Hence, a quantitative understanding of the observed contrast is not easily accessible. We can, however, interpret the contrast in our data qualitatively since we typically see the same characteristic checkerboard pattern in our experiments.

We ascribe the modulations within amplitude and phase signal to the three hydration layers above the calcite surface, in line with previous experimental findings [17, 18]. Although the three minima are easily identified in figures $3(\mathrm{c}),(\mathrm{d})$, a correct assignment of hydration layers requires knowledge about the vertical position of the calcite surface within the $2 \mathrm{D}$ slices. Typical criteria for physical contact between cantilever tip and sample surface are usually derived from analysing the damping signal (FM-AFM) [40] or the static deflection signal (AM-AFM) [41]. To gain detailed insight, we evaluated the relationship between the static deflection signal and the oscillation amplitude signal.

The behavior of the static deflection signal and of the oscillation amplitude signal as a function of the tip-sample distance can be seen in figure 4. This experiment was performed at a temperature of $301 \mathrm{~K}$ with a free amplitude of $0.5 \mathrm{~nm}$, an amplitude setpoint of 0.9 and a wide modulation range of $\pm 3.1 \mathrm{~nm}$.

According to Burnham et al [41], three different regimes (I to III in figure 4) can be distinguished when the tip approaches the sample. Far away from the surface and regardless of the medium, both amplitude and static deflection are independent of the tip-sample distance (regime I). In regime $\mathrm{I}$, the static deflection is zero. When the tip starts to interact with the surface (regime II) the amplitude decreases. In regime II, the static deflection behaves differently in water and in air. In air the static deflection remains zero within regime II. In water the static deflection starts to increase as 


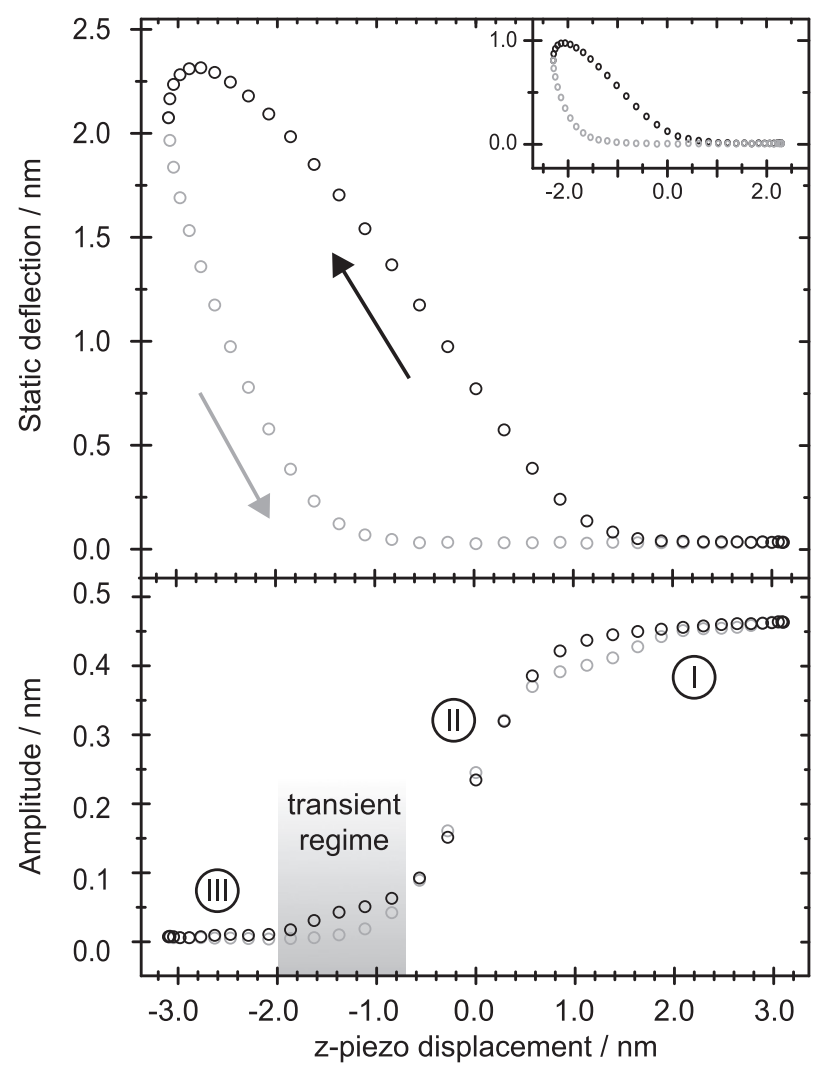

Figure 4. Static deflection and oscillation amplitude curves as a function of the z-piezo displacement. The direction of the tip-sample movement is indicated by arrows; approach and retract are shown in black and gray, respectively. The curves are averaged over 120 single curves, i.e., an entire scan line. The inset shows a static deflection curve from the same experiment at smaller modulation range.

soon as the amplitude decreases due to, e.g., interaction between tip and hydration layers. In regime III, upon further approach to the sample, the amplitude remains at a constant low value, while the static deflection increases linearly according to the increasing z-piezo displacement. We can see the same behavior in our data (figure 4). The fast data acquisition (1.36 ms per curve in figure 4) leads to a notable hysteresis in the static deflection between approach and retract. The sinusoidal fashion of the $\mathrm{z}$-movement leads to a reduction of this hysteresis around the turning points since the z-modulation speed is smallest there. This is the origin for the (non-physical) decrease of static deflection around the point of closest approach. For the following analysis we concentrate on the approach part of the static deflection curve.

While in air the onset of the static deflection coincides with the collapse of the amplitude, such a clear identification criterion for the surface position is not available in liquids, because the static deflection increases in regime II and in regime III. Nevertheless, it is fair to expect the interaction in regime II to be dominated by the interaction between tip and solution while in regime III the interaction between tip and surface is dominant. The static deflection curve (figure 4) shows a smooth transition from a smaller slope in regime II towards a larger slope in regime III. These different slopes are easier to see when comparing static deflection curves of different modulation range, i.e., differing interaction regime. The inset in figure 4 shows the static deflection curve for a smaller modulation range. The slope of this curve is somewhat smaller since the tip does not enter regime III in this case. It is the absence of a clear kink in the static deflection curve between regime II and regime III that renders the static deflection alone insufficient as criterion for the assignment of the surface position. This is the very reason why we base our criterion on the static deflection and the amplitude-distance curve.

As indicated in figure 4, the transient regime between regime II and regime III may spread over a considerable tipsample distance range. The amplitude, however, does not change a lot within this transient regime. For the example given in figure 4 , the transition between liquid and surface interaction is located at an amplitude of $(0.05 \pm 0.03) \mathrm{nm}$. Although the border between regime II and III is hard to locate precisely in $z$, the determination of the surface position via this method is good enough to assign the hydration layers without any doubt within the amplitude slices. However, this determination is certainly not precise enough to allow for measuring the separation between surface and first hydration layer.

Based on theoretical work, the first hydration layer is assigned to strongly bound water molecules above the calcium ions, while the second layer is attributed to water molecules forming hydrogen bonds towards the surface carbonate groups. Therefore, we expect well-ordered hydration layers in registry with the surface periodicity but with differing contrast. In figure 5, a further AM-AFM measurement of calcite in aqueous solution is presented. Here, the temperature was $304 \mathrm{~K}$, the free amplitude was $0.3 \mathrm{~nm}$, and we used an amplitude setpoint of 0.7 and a modulation range of $\pm 0.5 \mathrm{~nm}$. In the $2 \mathrm{D}$ slice along the [01.0] surface direction (figure 5(a)) the same checkerboard pattern is visible as in figure 3(a). With the criterion described above (see figure 4), we can distinguish the calcite surface from the hydration layers. Besides the strongly bound first and second layer, we can clearly identify a third hydration layer. However, the comparison of the data shown in figures 3(a) and 5(a) reveals the strong dependence of the visibility of this third layer on the tip and imaging conditions.

The $z$-axis in our 3D maps corresponds to the change in tip-sample distance, which only results from the sinusoidal movement of the z-piezo. The true tip-sample distance change, however, is determined by many more effects, e.g., tip and surface relaxations. Therefore, absolute tip-sample distances are experimentally not accessible and only apparent distances can be measured. For the comparison with recent experimental and theoretical work, we measured the apparent distance between the hydration layers in 2D slices like those shown in figures 3(a) and 5(a). We determined apparent distances of about $1.4 \AA$ between the first and the second hydration layer and $1.5 \AA$ between the second and the third hydration layer. Imada and co-workers [17] have measured distances of $1.4 \AA$ (between first and second layer) and $1.6 \AA$ 

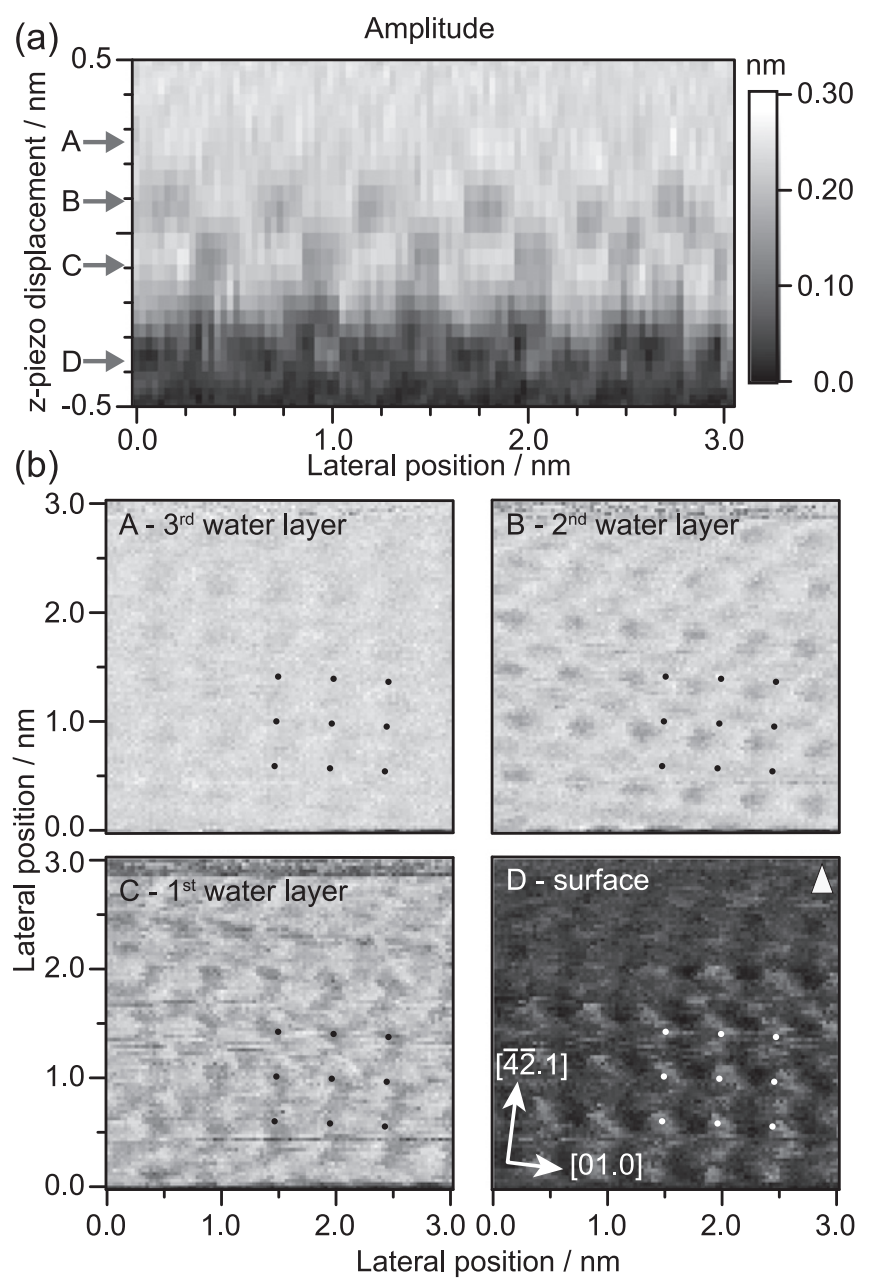

Figure 5. Corresponding 2D slices (a) along the [01.0] surface direction and (b) parallel to the surface extracted from the same 3D map. The positions of the images in (b) are indicated by arrows labeled A-D. All slices show amplitude data depicted in the same color scale. An array of black (white) dots marks identical lattic points in $\mathrm{A}-\mathrm{C}(\mathrm{D})$.

(between second and third layer). Kerisit and co-workers have calculated the separation between hydration layers starting from the first layer as follows: $1.0 \AA$, 1.8 $\AA, 2.8 \AA$ [19]. Thus, the apparent distances obtained here compare well with previous experimental and theoretical work.

We extracted 2D slices parallel to the surface (equivalent to constant-height images) at different tip-sample distances from the same 3D map (figure 5(b)). This allows for a direct comparison of the calcite surface with the first, second and third hydration layer. As expected, all four images in figure 5(b) show a periodic, rectangular structure commensurate to the surface unit cell of calcite (10.4) as introduced in figure 1. This observation illustrates the strong templating effect of the calcite surface causing the formation of ordered hydration layers. From the images in figure 5(b) it is evident why 3D mapping is clearly superior to standard AFM imaging: from a single AFM image alone it is impossible to deduce whether the surface or one of the hydration layers is imaged.

\section{Summary and conclusion}

We present a straightforward implementation for 3D data collection using a commercial AFM. Our routine is based on combining the easy-to-use AM-AFM technique with reliable high-speed 3D data acquisition for hydration layer mapping. We address the issue of free amplitude variations due to the drifting 'forest of peaks'. We solved this problem by periodically adjusting the excitation amplitude during data acquisition. The performance of our set-up is demonstrated by presenting 3D data taken on the calcite (10.4) surface in aqueous solution. Our data reproducibly reveals hydration layers with distinct patterns at $\AA$ resolution. We can clearly distinguish three hydration layers with a periodic structure commensurate to the calcite surface. The observed checkerboard pattern as well as the spacing between the hydration layers is in excellent agreement with previous experimental and theoretical results.

\section{Acknowledgments}

CM is grateful to Dr Wolfgang Harneit for sharing his detailed knowledge of the Igor Pro Software.

\section{References}

[1] Meldrum F C 2003 Int. Mater. Rev. 48 187-224

[2] Stipp S L S, Eggleston C M and Nielsen B S 1994 Geochim. Cosmochim. Acta 58 3023-33

[3] Ohnesorge F and Binnig G 1993 Science 260 1451-6

[4] Rachlin A L, Henderson G S and Goh M C 1992 Am. Mineral. 77 904-10

[5] Raina G, Gauldie R W, Sharma S K and Helsley C E 1994 Ferroelectr. Lett. Sect. 17 65-72

[6] Liang Y, Lea A S, Baer D R and Engelhard M H 1996 Surf. Sci. 351 172-82

[7] Rode S, Oyabu N, Kobayashi K, Yamada H and Kühnle A 2009 Langmuir 25 2850-3

[8] McEvoy A L, Stevens F, Langford S C and Dickinson J T 2006 Langmuir 22 6931-8

[9] Hillner P E, Manne S, Gratz A J and Hansma P K 1992 Ultramicroscopy 42 1387-93

[10] Dove P M, Hochella Jr and Michael F 1993 Geochim. Cosmochim. Acta 57 705-14

[11] Gratz A J, Hillner P E and Hansma P K 1993 Geochim. Cosmochim. Acta 57 491-5

[12] Britt D W and Hlady V 1997 Langmuir 13 1873-6

[13] Schreiber M, Eckardt M, Klassen S, Adam H, Nalbach M, Greifenstein L, Kling F, Kittelmann M, Bechstein R and Kühnle A 2013 Soft Matter 9 7145-9

[14] Geissbühler P, Fenter P, DiMasi E, Srajer G, Sorensen L B and Sturchio N C 2004 Surf. Sci. 573 191-203

[15] Fenter P and Sturchio N C 2012 Geochim. Cosmochim. Acta $9758-69$

[16] Heberling F, Trainor T P, Lützenkirchen J, Eng P, Denecke M A and Bosbach D $2011 \mathrm{~J}$. Colloid Interface Sci. 354 843-57

[17] Imada H, Kimura K and Onishi H 2013 Langmuir 29 10744-51 
[18] Kobayashi N, Reischl B, Foster A S, Asakawa H and Fukuma T 2013 Conf. Contribution on the 16th Int. Conf. on Non-Contact Atomic Force Microscopy (Maryland, USA)

[19] Kerisit S and Parker S C 2004 Chem. Commun. 52-53

[20] Perry T D, Cygan R T and Mitchell R 2007 Geochim. Cosmochim. Acta 71 5876-87

[21] Raiteri P, Gale J D, Quigley D and Rodger P M 2010 J. Phys. Chem. C 114 5997-6010

[22] Reischl B, Watkins M and Foster A S 2013 J. Chem. Theory Comput. 9 600-8

[23] Schwarz A, Hölscher H, Langkat S M, Wiesendanger R, Koenraad P M and Kemerink M 2003 AIP Conf. Proc. 696 $68-78$

[24] Albers B J, Schwendemann T C, Baykara M Z, Pilet N, Liebmann M, Altman E I and Schwarz U D 2009 Nanotechnology 20264002

[25] Rahe P, Schütte J, Schniederberend W, Reichling M, Abe M, Sugimoto Y and Kühnle A 2011 Rev. Sci. Instrum. 82 063704-7

[26] Kimura K, Ido S, Oyabu N, Kobayashi K, Hirata Y, Imai T and Yamada H 2010 J. Chem. Phys. 132194705

[27] Hiasa T, Kimura K, Onishi H, Ohta M, Watanabe K, Kokawa R, Oyabu N, Kobayashi K and Yamada H 2010 J. Phys. Chem. C 114 21423-6

[28] Suzuki K, Oyabu N, Kobayashi K, Matsushige K and Yamada H 2011 Appl. Phys. Express 4125102
[29] Nishioka R, Hiasa T, Kimura K and Onishi H 2013 J. Phys. Chem. C 117 2939-43

[30] Hiasa T, Kimura K and Onishi H 2012 Phys. Chem. Chem. Phys. 14 8419-24

[31] Asakawa H, Yoshioka S, Nishimura K and Fukuma T 2012 ACS Nano 6 9013-20

[32] Fukuma T, Higgins M J and Jarvis S P 2007 Biophys. J. 92 3603-9

[33] Hiasa T, Kimura K and Onishi H 2012 J. Phys. Chem. C 116 26475-9

[34] Hiasa T, Kimura K and Onishi H 2012 Colloids Surf. A 396 203-7

[35] Fukuma T, Ueda Y, Yoshioka S and Asakawa H 2010 Phys. Rev. Lett. 104016101

[36] Schäffer T E, Cleveland J P, Ohnesorge F, Walters D A and Hansma P K 1996 J. Appl. Phys. 80 3622-7

[37] Lübbe J, Temmen M, Rahe P, Kühnle A and Reichling M 2013 Beilstein J. Nanotechnol. 4 227-33

[38] Bresenham J E 1965 IBM Syst. J. 4 25-30

[39] Watkins M and Shluger A L 2010 Phys. Rev. Lett. 105196101

[40] Labuda A, Kobayashi K, Suzuki K, Yamada H and Grütter P 2013 Phys. Rev. Lett. 110066102

[41] Burnham N A, Behrend O P, Oulevey F, Gremaud G, Gallo P J, Gurdon D, Dupas E, Kulik A, Pollock H and Briggs G 1997 Nanotechnology 867 